ORIGINAL ARTICLE

\title{
In Vitro Antifungal Susceptibility of Environmental Isolates of Cryptococcus spp. from the West Region of Cameroon
}

\author{
William Dongmo ${ }^{1}$, Frederick Kechia ${ }^{2,3}$, Roland Tchuenguem ${ }^{1}$, Claude \\ Nangwat $^{1}$, Iwewe Yves ${ }^{2}$, Jules-Roger Kuiate ${ }^{1}$, Jean Paul Dzoyem ${ }^{1}$
}

\begin{abstract}
BACKGROUND: Cryptococcus neoformans is responsible of cryptococcosis, a life-threatening infection that affects healthy and immunocompromised individuals. It is the first cause of adult acute meningitis in some sub-Saharan African countries with a mortality rate of about $100 \%$ in cases of inappropriate therapy. This study aimed at examining the occurrence and the antifungal patterns of Cryptococcus isolates from pigeon droppings and bat guanos in the west region of Cameroon.

METHODS: A total of 350 samples were randomly collected from three selected localities of west region of Cameroon. The identification was performed based on capsule production assessed by Indian ink preparation. Additional tests performed were urea broth, glycine and tryptophan assimilation tests. The antifungal susceptibility test was performed by the broth microdilution method.

RESULTS: Mycological analysis led to the identification of 98 isolates, of which 57 isolates of $C$. neoformans var. gattii and 41 isolates of $C$. neoformans var. neoformans. All the isolates showed resistance to antifungals tested except nystatin which showed MIC mean values ranging between 0.5 $\mu \mathrm{g} / \mathrm{mL}$ and $0.65 \mu \mathrm{g} / \mathrm{mL}$.
\end{abstract}

CONCLUSION: The prevalence of $C$. neoformans in pigeons and bats excreta in the west region of Cameroon is $28.57 \%$. C. neoformans var. gattii and C. neoformans var. neoformans are the main serotypes. Isolates found to be resistant to fluconazole and ketoconazole. Our results emphasize the need for further study on the molecular epidemiology in comparison with clinical isolates.

KEYWORDS: Cryptococcus neoformans, bird excreta, antifungal susceptibility

DOI: http://dx.doi.org/10.4314/ejhs.v26i6.8

\section{INTRODUCTION}

Cryptococcus neoformans is an encapsulated, ubiquitous environmental yeast that causes cryptococcosis, a potentially serious disease that affects healthy and immunocompromised individuals, especially patients with AIDS (1). It is actually the first cause of adult acute meningitis in some sub-Saharan African countries with a mortality rate of about $100 \%$ in cases of inappropriate therapy, and about $20 \%$ to $30 \%$ in cases of antifungal treatment (1,2). The aetiological agents of cryptococcosis comprise four serotypes grouped into two major species, namely, Cryptococcus gattii (serotypes B and C) and Cryptococcus neoformans, (serotype A and D) (3). These two species differ in their biochemical characteristics especially their amino acids assimilation ability and clinical characteristics $(4,5)$. C. neoformans var. grubii is mostly incriminated in infections with immunocompromised hosts while $C$. neoformans var. gattii predominantly affects

\footnotetext{
${ }^{1}$ Laboratory of Microbiology and Antimicrobial Substances (LAMAS), Department of Biochemistry, Faculty of Sciences, University of Dschang, Cameroon

${ }^{2}$ Medical/Clinical Mycology Laboratory, Faculty of Medicine and Biomedical Sciences, University of Yaounde I, Cameroon

${ }^{3}$ Department of Biomedical Sciences, Faculty of Health Sciences, University of Bamenda, Cameroon

Corresponding Author: Jean Paul Dzoyem, Email: jpdzoyem@yahoo.fr
} 
immunocompetent hosts $(6,7,8)$. They also differ in their ecological niches and geographical distribution. Globally, the distribution of Cryptococcus species in nature is very large and is especially associated to decaying wood of certain species of trees, fruits and bird droppings, particularly pigeon droppings (9). $C$. neoformans is commonly isolated from bird excreta while $C$. gattii has been isolated mainly from samples of eucalyptus and other trees around the world (10). As infection by $C$. neoformans occurs through inhalation of propagules from the environment, bird excreta as a source of infectious propagules could play a central role in the transmission of $C$. neoformans from the environment to humans. Therefore, bird excreta appear as a dangerous reservoir and potential source of inhaled $C$. neoformans. In the west region of Cameroon, pigeons are domesticated and therefore have a very close proximity to humans. In addition, there is an increasing presence of bats in urban areas, colonizing many tree species. Considering the incidence of human cryptococcosis in Cameroon and the fact that birds are becoming more close to humans in some regions, this study was undertaken to determine the prevalence of $C$. neoformans in pigeon droppings and bat guanos in three localities of the west region of Cameroon. Furthermore, the antifungal susceptibility of the isolates was evaluated against four commonly used antifungals.

\section{MATERIALS AND METHODS}

Study area and sample collection: The West Region is $13,892 \mathrm{~km}^{2}$ of territory located in the central-western portion of the Republic of Cameroon. Its population is estimated to be $1,834,800$. Based on the high presence of bats and pigeons, three localities including Bafoussam, Dschang and Santchou were selected for the study. Isolation and identification of Cryptococcus species: Samples were randomly collected. Dry samples were collected with spatulas and placed in identified clean plastic bags. Wet samples were collected with a sterile cotton-tipped swab moistened in sterile saline solution $(0.85 \% \mathrm{NaCl})$ supplemented with chloramphenicol $(10 \mu \mathrm{g} / \mathrm{ml})$ and placed in a test tube with $3 \mathrm{ml}$ of the same solution. The collected samples were transported in sterilized plastic bags. The samples were processed following the method described by Soogarun (11), but with slight modifications on the isolation procedure. One gram of each sample was dissolved in $9 \mathrm{~mL}$ of sterile physiological saline $(0.9 \%$ aqueous $\mathrm{NaCl})$ containing $0.4 \mathrm{mg} / \mathrm{mL}$ of chloramphenicol, vortexed vigorously for 1-3 min and filtered through sterile gauze. After 10 minutes, $50 \mu \mathrm{L}$ of supernatant was streaked onto Sabouraud's dextrose agar plate containing 0.05 $\mathrm{mg} / \mathrm{mL}$ of chloramphenicol. Plates were then incubated at $37{ }^{\circ} \mathrm{C}$ for 3-21 days. Creamed round brown yeast-like colonies of suspected $C$. neoformans detected were subcultured on new Sabouraud dextrose agar tubes to obtain single colonies.

Colony morphology and microscopic observation of the presence of capsule in India ink preparation were performed. Urease enzyme production was assessed on prepared indole-urea agar culture medium following the manufacturer's protocol. For the biochemical tests, the auxanogram technique was used to differentiate, identify and confirm the specie variety. In fact, on glycine and tryptophan assimilation testing composed media based on the fact that only $C$. neoformans var. gattii isolates are able to use glycine and tryptophan as sole sources of nitrogen $(4,5)$. For the test, $175 \mu \mathrm{L}$ of each composed media (media composition: glucose $20 \mathrm{~g} / \mathrm{L}$, glycine $20 \mathrm{~g} / \mathrm{L}$ or tryptophan $4 \mathrm{~g} / \mathrm{L}$ and Chloramphenicol $0.05 \mathrm{~g} / \mathrm{L}$ ) was introduced in 96 well microtiter plates and $25 \mu \mathrm{L}$ of each solution prepared in concentrations corresponding to 0.5 on the Mac-Farland scale, was added. The plates were then incubated at 37 ${ }^{\circ} \mathrm{C}$ for 48 to 72 hours, and the growth was recorded. C. neoformans KN99a (serotype A) was used as reference strain for control.

Antifungal susceptibility testing: In addition to the reference clinical strains $C$. neoformans $\mathrm{KN} 99 \alpha$, ten isolates of each variety were randomly selected and tested for their antifungal susceptibility to four commonly used antifungals: namely, nystatin, amphotericin $\mathrm{B}$, fluconazole and ketoconazole. The minimum inhibitory concentration was determined as recommended by the NCCLS (12). MIC values were interpreted as described by Lozano-Chiu et al. and Nguyen and Yu respectively $(13,14)$ (Table 1). 
Table 1: Minimum inhibitory concentration (MIC) values interpretation

\begin{tabular}{lccc}
\hline \multirow{1}{*}{ Antifungals } & \multicolumn{3}{c}{ MIC range $(\mu \mathrm{g} / \mathrm{mL})$} \\
\cline { 2 - 4 } & Sensible & $\begin{array}{c}\text { Intermediately } \\
\text { susceptible }\end{array}$ & Resistant \\
\hline Fluconazole & $\leq 8$ & $16-32$ & $\geq 64$ \\
AmphotericinB & $\leq 1$ & $2-4$ & $>4$ \\
Ketoconazole & $\leq 0,125$ & $0.25-0.5$ & $\geq 1$ \\
Nystatin & $\leq 1$ & $2-4$ & $>4$ \\
\hline
\end{tabular}

\section{RESULTS}

A total of 350 samples were collected over a period of eight months, from April to November 2015. They were made up of 200 samples of pigeon droppings and 150 samples of bat guanos (Figure 1). From the 350 samples collected, 103 yeast-like Cryptococcus were isolated among which 101 showed capsules in the India ink preparation. The 101 India ink positive isolates subjected to urease enzyme test revealed 100 positive and 01 negative. Therefore, 100 isolates were confirmed as Cryptococcus neoformans isolates (Table 2). Glycine and tryptophan assimilation results showed that, among the 100 isolates positive to urease, 57 were of the Cryptococcus gattii variety, while 41 were of the Cryptococcus neoformans var neoformans (Cryptococcus neoformans) variety and 02 of the isolates remained undetermined (Table 3 ). The antifungal results are presented in (Table 4). Compared to the reference clinical strain (MICs ranged from $4 \mu \mathrm{g} / \mathrm{mL}$ to $32 \mu \mathrm{g} / \mathrm{mL}$ ), the antifungal susceptibility results showed high resistance of isolates to azole antifungals. The MICs values ranged from $16 \mu \mathrm{g} / \mathrm{mL}$ to $>256 \mu \mathrm{g} / \mathrm{mL}$ and from 8 $\mu \mathrm{g} / \mathrm{mL}$ to $64 \mu \mathrm{g} / \mathrm{mL}$ for fluconazole and ketoconazole respectively.

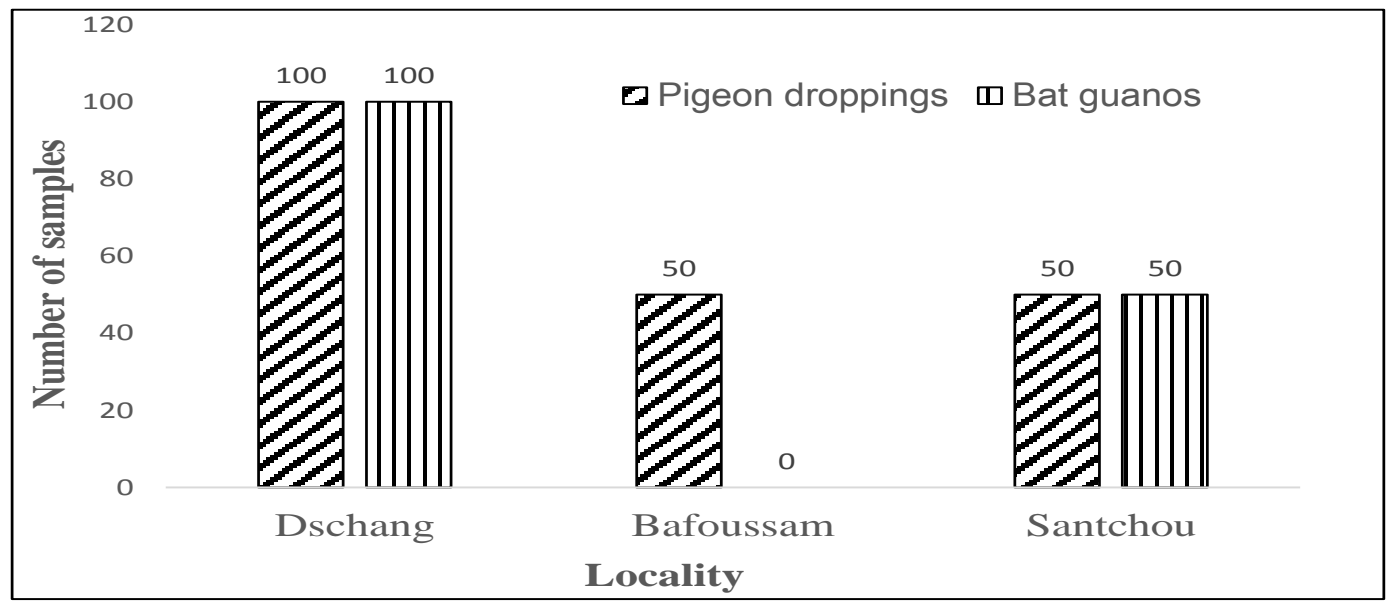

Figure 1: Distribution of samples according to the collection areas

Table 2: Results of culture and microscopic observation in India ink

\begin{tabular}{llcccccccc}
\hline & $\begin{array}{l}\text { Types of } \\
\text { excreta }\end{array}$ & $\begin{array}{c}\text { Culture } \\
(\mathbf{n = 3 5 0})\end{array}$ & $\mathbf{\%}$ & $\begin{array}{c}\text { India ink } \\
(\mathbf{n = 1 0 3})\end{array}$ & $\mathbf{\%}$ & $\begin{array}{c}\text { Urease test } \\
(\mathbf{n = 1 0 1})\end{array}$ & $\mathbf{\%}$ & Total & \% \\
\hline \multirow{3}{*}{ Positive } & PD & 56 & 16 & 56 & 54.37 & 55 & 54.45 & $\mathbf{5 5}$ & 55 \\
& BG & 47 & 13.42 & 45 & 43.68 & 45 & 44.55 & $\mathbf{4 5}$ & 45 \\
& Total & $\mathbf{1 0 3}$ & $\mathbf{2 9 . 4 2}$ & $\mathbf{1 0 1}$ & $\mathbf{9 8 , 0 5}$ & $\mathbf{1 0 0}$ & 99 & $\mathbf{1 0 0}$ & 100 \\
& PD & 144 & 41.14 & 0 & 0 & 1 & 1 & & \\
Negative & BG & 103 & 29.44 & 2 & 1.95 & 0 & 0 & & \\
& Total & 247 & $\mathbf{7 0 . 5 8}$ & $\mathbf{2}$ & $\mathbf{1 . 9 5}$ & $\mathbf{1}$ & 1 & & \\
\hline
\end{tabular}


Table 3: Glycine and tryptophan assimilation results and interpretation

\begin{tabular}{lcccccc}
\hline & \multicolumn{2}{c}{ Positive } & Total & \multicolumn{2}{c}{ Negative } & Total \\
\cline { 2 - 7 } Amino acid & $\begin{array}{c}\text { PD } \\
(\mathbf{n = 5 5})\end{array}$ & $\begin{array}{c}\text { BG } \\
(\mathbf{n = 4 5})\end{array}$ & & $\begin{array}{c}\text { PD } \\
(\mathbf{n = 5 5})\end{array}$ & $\begin{array}{c}\text { BG } \\
(\mathbf{n = 4 5})\end{array}$ & \\
\hline Gly & 24 & 18 & 42 & 31 & 27 & 58 \\
Trp & 23 & 19 & 42 & 32 & 26 & 58 \\
Trp+Gly & 23 & 18 & $\mathbf{4 1}$ & 31 & 26 & $\mathbf{5 7}$ \\
Interpretation & & & & & & \\
Cn var neoformans & & & & 31 & 26 & $\mathbf{5 7}$ \\
Cn var gattii & 23 & 18 & $\mathbf{4 1}$ & & & \\
Undetermined & 1 & 1 & $\mathbf{2}$ & & & \\
Cryptococcus neoformans; $\mathrm{PD}=$ Pigeon dropping; BG=Bat guano; Gly=glycine; Trp=tryptophane
\end{tabular}

Table 4: Antifungal susceptibility of isolates and a reference clinical strain determined by the minimum inhibitory concentration (MIC).

\begin{tabular}{|c|c|c|c|c|}
\hline \multirow{3}{*}{$\begin{array}{l}\text { Antifungals } \\
\text { nof }\end{array}$} & & \multicolumn{3}{|c|}{$\operatorname{MIC}(\mu \mathrm{g} / \mathrm{mL})$} \\
\hline & & \multicolumn{2}{|c|}{$\begin{array}{c}\text { Environmental } \\
\text { isolates }\end{array}$} & \multirow{2}{*}{$\begin{array}{c}\begin{array}{c}\text { Reference } \\
\text { clinical } \\
\text { strain }\end{array} \\
\text { Cn } K N 99 \alpha\end{array}$} \\
\hline & & $\begin{array}{c}\text { Cn var } \\
\text { neoformans } \\
(\mathrm{n}=10)\end{array}$ & $\begin{array}{c}\text { Cn var } \\
\text { gattii } \\
(\mathrm{n}=10)\end{array}$ & \\
\hline \multirow[t]{2}{*}{ Fluconazole } & Range & $64->256$ & $16->256$ & 2 \\
\hline & Mean & - & - & \\
\hline \multirow[t]{2}{*}{ Ketoconazole } & Range & $16-64$ & $8-64$ & 1 \\
\hline & Mean & 36.8 & 27.2 & \\
\hline \multirow[t]{2}{*}{ Amphotericin B } & Range & $4-8$ & $64-128$ & 2 \\
\hline & Mean & 4.6 & 89.6 & \\
\hline \multirow[t]{2}{*}{ Nystatin } & Range & $0.125-0.5$ & $0.5-1$ & 0.5 \\
\hline & Mean & 0.35 & 0.65 & \\
\hline
\end{tabular}

Cn $=$ Cryptococcus neoformans, $\mathrm{n}=$ number of isolates

\section{DISCUSSION}

The incidence of opportunistic fungal infections has increased in recent years and is considered as an important public health problem. Several studies have shown that $C$. neoformans remains viable in the dried excrement of birds (15). Therefore bird excreta appear as a dangerous reservoir and potential source of $C$. neoformans infection. In this regard, 350 samples of pigeon droppings and bat guanos were collected in the west region of Cameroon and the isolates obtained were examined for their antifungal susceptibility.

In this study, we observed an occurrence of C. neoformans in 100 out of the 350 samples analysed, indicating an incidence of $28.57 \%$ of this yeast in the studied region. This result confirms the presence of this yeast in the environment, in the west region of Cameroon. Similar studies carried out in Nigeria and Jordan showed prevalences of $22.0 \%$ (39/177) and $33.3 \%$ $(336 / 1009)$ respectively $(16,17)$. However, in the above studies, the authors used only pigeon dropping and materials under canopies of eucalyptus trees for isolation, not bat guanos. In our study, the number of isolates obtained from pigeon droppings (55\%) was higher than those recovered from bat guanos (45\%), confirming pigeon droppings as an important reservoir of $C$. neoformans. Previous studies have described pigeon droppings as the main source of $C$. neoformans isolation (18). Nevertheless, with $45 \%$ of $C$. neoformans recovered from bat guanos, bats may also play an important role in the 
epidemiology of cryptococcosis through their migratory characters. The presence of the two main varieties of $C$. neoformans, namely $C$. neoformans var. neoformans and $C$. neoformans var. gattii in the west region of Cameroon confirm the geographical distribution of $C$. neoformans as described in several previous studies $(15,5,4,19)$. In fact, most of these previous studies showed that $C$. neoformans var. neoformans has a worldwide distribution while $C$. neoformans var. gattii is associated with decaying wood in tropical and sub-tropical regions of the world. Nevertheless, Overy et al (20) identified the presence of Cryptococcus gattii in Canada showing that this pathogen colonizes other parts of the world.

All the isolates tested showed resistance to fluconazole, ketoconazole and amphotericin B antifungals. Such a pattern of susceptibility from environmental $C$. neoformans isolates was not found in the literature. Most studies reported that environmental isolates of $C$. neoformans were susceptible to these antifungals $(21,22)$. Gutch et al (23), using the microdilution method, verified the susceptibility profile of clinical and environmental isolates of Cryptococcus neoformans and Cryptococcus gattii in Jabalpur, a city of Madhya Pradesh in Central India. Although low sensitivity of environmental isolates to fluconazole has been demonstrated by Rossi et al (24), these results may draw the attention of clinician researchers on a prospective emergence of resistant clinical isolates since contamination can easily occur by inhalation of propagules from the environment. Contrary to amphotericin B, our isolates appeared to be susceptible to nystatin which is also an antifungal belonging to the polyene class.

This study revealed that the prevalence of $C$. neoformans in the excreta of pigeons and bats in the west region of Cameroon is $28.57 \%, C$. neoformans var. gattii and $C$. neoformans var. neoformans being the two main representative serotypes. Isolates were found to be resistant to fluconazole and ketoconazole. Considering the incidence of human cryptococcosis in Cameroon, especially in HIV patients, and the fact that pigeons and bats are widely spread birds in these localities, our results emphasize the need for further studies on the molecular epidemiology in comparison with clinical isolates.

\section{ACKNOWLEDGMENT}

JP Dzoyem is thankful to "The World Academy of Sciences (TWAS) for the advancement of science in developing countries" for supporting this work through the TWAS Research Grant Agreement No:11-128 $\mathrm{RG} / \mathrm{CHE} / \mathrm{AF} / \mathrm{AC}$ _UNESCO

FR:3240262681.

\section{REFERENCES}

1. Dromer F, Lortholary O. Cryptococcose. EMC-Maladies Infectieuses, 2003; 8-613-A$10,10$.

2. Gullo FP, Rossi SA, Sardi Jde C, Teodoro VL, Mendes-Giannini MJ, Fusco-Almeida AM. Cryptococcosis: epidemiology, fungal resistance, and new alternatives for treatment. Eur J Clin Microbiol Infect Dis. 2013; 32(11):1377-91.

3. Kwon-Chung KJ, Fraser JA, Doering TL, Wang Z, Janbon G, Idnurm A, Bahn YS. Cryptococcus neoformans and Cryptococcus gattii, the etiologic agents of cryptococcosis. Cold Spring Harb Perspect Med. 2014; 4(7): a019760.

4. Chan MY, Tay ST. Enzymatic characterisation of clinical isolates of Cryptococcus neoformans, Cryptococcus gattii and other environmental Cryptococcus spp. Mycoses. 2010; 53(1):26-31

5. Chaskes S, Frases S, Cammer M, Gerfen G, Casadevall A. Growth and pigment production on D-Tryptophan medium by Cryptococcus gattii, Cryptococcus neoformans, and Candida albicans . J Clin Microbiol. 2008; 46: 255-264.

6. Mseddi F, Sellami A, Jarboui MA, Sellami H, Makni F, Ayadi A. First environmental isolations of Cryptococcus neoformans and Cryptococcus gattii in Tunisia and review of published studies on environmental isolations in Africa. Mycopathologia. 2011; 171(5):35560.

7. Chowdhary A, Rhandhawa HS, Prakash A, Meis JF. Environmental prevalence of Cryptococcus neoformans and Cryptococcus gattii in India: an update. Crit Rev Microbiol. 2012; 38(1):1-16.

8. Idnurm A, Lin X. Rising to the challenge of multiple Cryptococcus species and the 
diseases they cause. Fungal Genet Biol. 2015; 78:1-6.

9. Mseddi F, Sellami A, Jarboui MA, Sellami H, Makni F, Ayadi A. First environmental isolations of Cryptococcus neoformans and Cryptococcus gattii in Tunisia and review of published studies on environmental isolations in Africa. Mycopathologia. 2011; 171(5):35560

10. Leite DP Jr, Amadio JV, Martins ER et al. Cryptococcus spp isolated from dust microhabitat in Brazilian libraries. $J$ Occup Med Toxicol. 2012; 7(1):11.

11. Soogarun S, Wiwanitkit V, Palasuwan A et al. Detection of Cryptococcus neoformans in bird excreta. Southeast Asian J Trop Med Public Health. 2006; 37(4):768-70.

12. National committee for Clinical Laboratory Standard (N.C.C.L.S). Reference method for broth dilution Antifungal susuptibility Testing of yeasts. Approved Standard M27A2. Wayne, USA. 17, 9, 2002.

13. Therese K L, Bagyalakshmi R, Madhavan HN, Deepa P. (2006). In-vitro susceptibility testing by agar dilution method to determine the minimum inhibitory concentrations of amphotericin B, fluconazole and ketoconazole against ocular fungal isolates. Indian J Med Microbiol. 2006; 24(4):273-9.

14. EUCAST. European Committee on Antimicrobial Susceptibility Testing Antifungal Agents Breakpoint tables for interpretation of MICs Version 6.1. 2013.

15. Casadevall A, Perfect, JR. Ecology of Cryptococcus neoformans. Washington, Asm Press, 1998.

16. Nweze E, Kechia F, Dibua U, Eze C, and Onoja U. Isolation of Cryptococcus neoformans from environmental samples collected in Southeastern Nigeria. Rev. Inst. Med. Trop. Sao Paulo. 2015; 57(4): 295-8.

17. Akram M, Sinasi T, Ahmet G, Mehmet A, and Levent. Cryptococcus neoformans varieties from material under the canopies of eucalyptus trees and pigeon dropping samples from four major cities in Jordan. Mycopathologia. 2004; 158: 195-199.

18. Li AS, Pan WH, Wu SX, Hideaki T, Guo NR, Shen YN, Lu GX, Pan RG, Zhu MC, Chen M, Shi WM, Liao WQ. Ecological surveys of the Cryptococcus species complex in China. Chin Med J (Engl). 2012; 125(3):511-6

19. Overy DP, McBurney S, Muckle A, Lund L, Lewis PJ, Strang R. Cryptococcus gattii VGIIb-like Variant in White-Tailed Deer, Nova Scotia, Canada. Emerg Infect Dis. 2016; 22(6):1131-3

20. Aade-Silva L, Ferreira-Paim K, Mora DJ, Da Silva PR, Andrade AA, Araujo NE, Pedrosa AL, Silva-Vergara ML. Susceptibility profile of clinical and environmental isolates of Cryptococcus neoformans and Cryptococcus gattii in Uberaba, Minas Gerais, Brazil. Med Mycol. 2013; 51(6): 635-640

21. Pedroso RS, Lavrador MAS, Ferreira JC, Candido RC, Maffei CML. Cryptococcus neoformans var. grubii Pathogenicity of environmental isolates correlated to virulence factors, susceptibility to fluconazole and molecular profile. Mem Inst Oswaldo Cruz. 2010; 105(8): 993-1000

22. Andrade-Silva L, Ferreira-Paim K, Mora DJ, Da Silva PR, Andrade AA, Araujo NE, Pedrosa AL, Silva-Vergara ML. Susceptibility profile of clinical and environmental isolates of Cryptococcus neoformans and Cryptococcus gattii in Uberaba, Minas Gerais, Brazil. Med Mycol. 2013; 51(6):635-40.

23. Gutch RS, Nawange SR, Singh SM, Yadu R, Tiwari A, Gumasta R, Kavishwar A. Antifungal susceptibility of clinical and environmental Cryptococcus neoformans and Cryptococcus gattii isolates in Jabalpur, a city of Madhya Pradesh in Central India. Braz J Microbiol. 2015; 46(4):1125-33.

24. Rossi SA, Trevijano-Contador N, Scorzoni L, Mesa-Arango AC, de Oliveira HC, Werther K, de Freitas Raso T, Mendes-Giannini MJ, Zaragoza O, Fusco-Almeida AM. Impact of Resistance to Fluconazole on Virulence and Morphological Aspects of Cryptococcus neoformans and Cryptococcus gattii Isolates. Front Microbiol. 2016; 7:153. 